\title{
Low-molecular-weight fucoidan protects endothelial function and ameliorates basal hypertension in diabetic Goto-Kakizaki rats
}

\author{
Wentong Cui ${ }^{1,5}$, Yuanyuan Zheng ${ }^{1,5}$, Quanbin Zhang ${ }^{2}$, Jing Wang ${ }^{2}$, Limin Wang ${ }^{1}$, Wenzhe Yang ${ }^{1}$, \\ Chenyang Guo ${ }^{1}$, Weidong Gao ${ }^{3}$, Xiaomin Wang ${ }^{4}$ and Dali Luo ${ }^{1}$
}

Endothelial dysfunction, characterized by impairment of endothelial nitric oxide synthase (eNOS) and nitric oxide (NO) bioavailability, has been implicated in diabetic cardiovascular pathogenesis. In this study, low-molecular-weight fucoidan (LMWF), which has multiple biological activities including anti-inflammatory and anti-oxidative properties, was investigated for its protective effect against endothelial dysfunction in Goto-Kakizaki type 2 diabetic rats. LMWF $(50,100$, or $200 \mathrm{mg} / \mathrm{kg} /$ day) or probucol $(100 \mathrm{mg} / \mathrm{kg} /$ day) were given to diabetic rats for 12 weeks. Basal blood pressure, acetylcholine- or flow-mediated relaxation of mesenteric and paw arteries, endothelium-dependent dilation of aorta, eNOS phosphorylation, and NO production were measured using laser Doppler flowmetry, force myograph, hematoxylin and eosin staining, western blot analysis, and an NO assay. We found that LMWF robustly ameliorated the basal hypertension and impairment of endothelium-dependent relaxation in the aorta, as well as mesenteric and paw arteries in diabetic rats. In addition, the reduction in eNOS phosphorylation at Ser1177, eNOS expression, and NO production because of diabetes were partially reversed by LMWF treatment. However, probucol, a lipid-modifying drug with antioxidant properties, displayed only mild effects. Moreover, LMWF induced, in a dose-dependent manner, endotheliumdependent vasodilation and eNOS phosphorylation at Ser1177 in normal aorta, and also promoted Ser1177 phosphorylation and NO synthesis in primary cultured vasoendothelial cells. Thus, these data demonstrate for the first time that fucoidan protects vasoendothelial function and reduces basal blood pressure in type 2 diabetes rats via, at least in part, preservation of eNOS function. Fucoidan is therefore a potential candidate drug for protection of endothelium in diabetic cardiovascular complications.

Laboratory Investigation (2014) 94, 382-393; doi:10.1038/labinvest.2014.12; published online 10 March 2014

KEYWORDS: diabetes; endothelium-dependent vasodilation; endothelial nitric oxide synthase; low-molecular-weight fucoidan; nitric oxide

Diabetic patients are at high risk of endothelial dysfunction and vascular lesions, because of multiple harmful stimuli, such as hyperglycemia, hyperlipidemia, and oxidative stress. These patients are susceptible to atherosclerosis, hypertension, and peripheral vascular disease. ${ }^{1,2}$ It is well established that endothelium-derived nitric oxide (NO) maintains vascular homeostasis and health through vasodilation and protection of vasculature from various damaging agents. ${ }^{3-5}$ Loss of NO induces increased activity of proinflammatory transcription factors, such as nuclear factor kappa B, resulting in expression of leukocyte adhesion molecules and production of chemokines and cytokines, which further promote endothelial inflammation and atherosclerosis. 5 ,6 Therefore, the endothelial dysfunction caused by imbalance of NO bioavailability in early-stage diabetes has been implicated as a sign or a predictor of a future cardiovascular event. ${ }^{6-8}$ Mechanistically, a disturbance in endothelial NO synthase (eNOS) activity, referred to as uncoupling of eNOS and downregulation of eNOS expression, ${ }^{2,9}$ has been identified as an important pathogenic

${ }^{1}$ Department of Pharmacology, Capital Medical University, Beijing, PR China; ${ }^{2}$ Institute of Oceanology, Chinese Academy of Sciences, Qingdao, PR China; ${ }^{3}$ Anesthesiology and Critical Care Medicine, Johns Hopkins University School of Medicine, Baltimore, MD, USA and ${ }^{4}$ Department of Physiology, Capital Medical University, Beijing, PR China

Correspondence: Professor D Luo, PhD, Department of Pharmacology, Capital Medical University, Street of Youanmenwai, \#10 Xitoutiao, Beijing 100069, PR China. E-mail: luodl@ccmu.edu.cn

${ }^{5}$ These authors contributed equally to this work.

Received 12 September 2013; accepted 11 December 2013 
factor in endothelial dysfunction and damage in type 1 and type 2 diabetes patients. ${ }^{3,7,10,11}$ In diabetes, eNOS uncoupling represents a functional shift of eNOS from production of NO to superoxide generation likely because of the reduction of the cofactor tetrahydrobiopterin and $\mathrm{L}$-arginine. ${ }^{10,11}$ Therefore, improvement of NO-related function represents a valuable pharmacological target for protecting endothelium and reducing the incidence of cardiovascular complication in diabetes patients. ${ }^{11,12}$ It is recognized that employing a single therapy to address hyperglycemia, hyperlipidaemia, or insulin resistance is not sufficient to protect against diabetic endothelial impairment and cardiovascular complications in diabetes patients. Alternatively, the addition of certain antioxidants, L-arginine, or protein kinase $\mathrm{C}$-inhibitors has been demonstrated to show additive benefits on endothelial function and cardiovascular protection. ${ }^{8,11-13}$

Fucoidan represents a family of L-fucose-enriched sulfated polysaccharides extracted from ubiquitous brown seaweed. This botanical polymer shares chemical analogies with heparin, a sulfated polysaccharide of animal origin, but exhibits lower anticoagulant activity. ${ }^{14}$ Algal fucoidans have been characterized as having a wide variety of biological activities in vivo and in vitro, including anti-inflammatory, antioxidative, and antiaggregative $e^{14,15}$ properties that all seem to be beneficial for alleviation of diabetic injury. In previous studies, we have successfully prepared and characterized a low-molecular-weight fucoidan (LMWF, $7000 \mathrm{Da}$ ) from $L$ aminaria japonica that shares similar biological activities with other fucoidans. ${ }^{16-18}$ In particular, LMWF has an advantage over the others in that it exerts antithrombotic effect without impacting coagulation, ${ }^{19}$ thus it has a lower hemorrhagic risk than other fucoidans. ${ }^{14,15}$ Therefore, we hypothesized that LMWF may protect against the endothelial and vascular disorders caused by diabetes. We conducted our experiments in Goto-Kakizaki (GK) rats for they manifest stable pathological features that resemble human type 2 diabetes with hyperglycemia, basal hypertension, endothelial dysfunction, and development of vascular complications. ${ }^{20,21}$ We evaluated in vivo effects of LMWF on blood pressure and local blood flow, ex vivo effects on endothelium-dependent vasodilation and eNOS expression, and in vitro effects of LMWF on eNOS phosphorylation in endothelial cells, and compared them with the effects of probucol, a lipidmodifying drug with powerful antioxidant and antiinflammatory properties used for prevention of type 2 diabetes mellitus. ${ }^{22-24}$ We found that LMWF profoundly protects endothelial function against the complications of diabetes.

\section{MATERIALS AND METHODS}

This study was approved by the Capital Medical University Animal Care and Use Committee, and all studies were conducted in accordance with 'Guide for the Care and Use of Laboratory Animals' adopted by the Beijing government and
'Guide for the Care and Use of Laboratory Animals' published by the US National Institutes of Health. ${ }^{25}$

\section{Materials}

LMWF (MW $=7000 \mathrm{Da}$ ) was supplied by the Institute of Oceanology, Chinese Academy of Sciences, and dissolved in distilled water. As previously described, LMWF was produced from the seaweed $L$. japonica commercially cultured in Qingdao, China, and analyzed as follows: fucose content 29.5\%; uronic acid content 7.5\%; and sulfate content $30.1 \%$. Its weight-average molecular weight $(\mathrm{Da})$ and numberaverage molecular weight $(\mathrm{Da})$ are $\sim 7000$ and $\sim 5300$, respectively. ${ }^{16,19}$ Probucol tablets were purchased from Qilu Pharmaceutical (Shandong, China). L-Phenylephrine, sodium nitroprusside dehydrate, acetylcholine chloride, and $\mathrm{N}^{\mathrm{G}}$ nitro-L-arginine methyl ester (L-NAME) were all purchased from Sigma-Aldrich. Anti-eNOS and anti-eNOS (phosphor S1177) antibodies were purchased from Abcam. Mouse antiGAPDH, $\alpha$-actin, goat anti-mouse, and goat anti-rabbit IgG HRPs were purchased from Santa Cruz. The BCA protein assay kit was purchased from Thermo. The rat total NO ELISA kit was purchased from BlueGene. All drugs were dissolved in saline, unless otherwise noted.

\section{Animals}

Twelve-week-old male GK rats and age-matched Wistar rats were purchased from SLAC Laboratory Animal (Shanghai, China) and reared in a standard experimental animal laboratory (12-h light/12-h dark cycle, room temperature $=21-22^{\circ} \mathrm{C}$, humidity $=60 \pm 5 \%$ ) with normal food and water given ad libitum. Wistar and GK rats were randomly divided into six groups: (1) Wistar control rats (normal group); (2) GK diabetic rats (DM group); $(3-5)$ GK rats treated with LMWF $(50,100$, and $200 \mathrm{mg} / \mathrm{kg}$ respectively, referred as LMWF1, LMWF2, and LMWF3 group), and (6) GK diabetic rats treated with probucol $(100 \mathrm{mg} / \mathrm{kg}$; probucol group). Drugs were given by intragastric administration between 0800 and 0900 hours every day for 12 weeks and equal volume of solvent was given to rats in normal and DM group. In addition, Wistar rats were also administered $100 \mathrm{mg} / \mathrm{kg} /$ day LMWF, which did not cause significant changes in all the test parameters compared with those in normal rats (data not shown).

Body weights were measured once a week, whereas blood pressure and plasma glucose were examined once a month. Oral glucose tolerance tests were performed at the outset of the experiment after rats fasted at least $12 \mathrm{~h}$. Blood was then taken from the caudal vein at $0,30,60,90,120,150$ and $180 \mathrm{~min}$ after oral administration of glucose $(2 \mathrm{~g} / \mathrm{kg})$. Plasma glucose concentration was measured with a glucometer (Roche Diagnostics, ACCU-CHEK Active, Germany). Blood pressure was monitored by a non-invasive CODA blood pressure monitoring system (CODATM, Kent Scientific, USA). After rats were warmed for $20-30 \mathrm{~min}$ at the heat chamber, which was set at about $37^{\circ} \mathrm{C}$, to make the 
pulsations of the tail artery detectable, the tail-cuff was inflated to a maximum pressure of $250 \mathrm{~mm} \mathrm{Hg}$ and blood pressure was detected using the system. Animals were acclimatized to this system for 3 successive days before the study to obtain reliable readings, and measurements were carried out at the same time (0900 - 1100 hours) each day in order to reduce the influence of circadian cycles on blood pressure. Blood samples were collected by carotid artery intubation technique and after clotting centrifuged at $1000 \mathrm{~g}$ for $15 \mathrm{~min}$ to collect the serum. The levels of cholesterol, triglyceride, high-density lipoprotein cholesterol (HDL-C), and low-density lipoprotein cholesterol (LDL-C) in the serum were determined using an automatic biochemistry analyzer (Beckman Coulter, USA). At the end of the experiment, rats were killed under sodium pentobarbital $(40 \mathrm{mg} / \mathrm{kg})$ anesthesia administered intraperitoneally.

\section{Measurement of Isolated Thoracic Artery Tension}

After rats were killed, the thoracic aorta was quickly and carefully removed, and adhering tissue was cleaned off with special care in ice-cold Krebs-Henseleit buffer (KHB, pH 7.40) containing (in mmol/l): $\mathrm{NaCl} 135.0, \mathrm{KCl} 4.7, \mathrm{CaCl}_{2} 2.5$, $\mathrm{MgSO}_{4} \cdot 7 \mathrm{H}_{2} \mathrm{O}$ 1.2, $\mathrm{KH}_{2} \mathrm{PO}_{4}$ 1.2, HEPES 5.0, glucose 11.0. To obtain endothelial-denuded aortic rings, we used appropriate size cotton swab to wipe the blood vessel lumen two to three times. The adjacent two 3- to 4-mm-long rings following the arcus aorta from each rat were used for vascular reactivity studies. Artery rings were installed on a pair of stainless-steel isosceles triangular brackets in $35-\mathrm{ml}$ organ chambers with $\mathrm{KHB}$ (at $37^{\circ} \mathrm{C}$ and bubbled with $95 \% \mathrm{O}_{2}$ and $5 \% \mathrm{CO}_{2}$ ). One bracket was at a fixed position; the other was connected to a force transducer for tension measurement (Radnoti organ bath system, USA) and recorded with a data-acquisition system (MP150, BIOPAC Systems, USA). The artery rings were stretched to a resting tension of $1.5 \mathrm{~g}$, and allowed to equilibrate for $90 \mathrm{~min}$, with the fresh buffer changed every $15-20 \mathrm{~min}$ before exposing to different drugs. Endothelium-dependent relaxation was measured by testing a single concentration $(10 \mu \mathrm{mol} / \mathrm{l})$ or concentration-dependent responses to cumulative addition of acetylcholine $\left(10^{-9}-10^{-5} \mathrm{~mol} / \mathrm{l}\right)$ in phenylephrine $(1 \mu \mathrm{mol} / \mathrm{l})$ preconstricted rings. Endothelium-independent relaxation to sodium nitroprusside was performed by the same method. In normal aorta, the effect of LMWF was also measured in phenylephrine pre-contracted endothelium-intact or denuded rings. Some rings were incubated with NOS blocker L-NAME $\left(10^{-4} \mathrm{~mol} / \mathrm{l}\right)$ for half an hour before addition of LMWF. Each relaxation response was expressed as a percentage of the contraction induced by phenylephrine.

\section{Histological Analysis of Sections of Aorta}

Appropriate $1.0 \mathrm{~cm}$ aorta was cut and immediately immersed in $4 \%$ paraformaldehyde for $24 \mathrm{~h}$, and then dehydrated with a graded series of increasing ethanol concentration. The whole vessel was embedded in paraffin and $5 \mu \mathrm{m}$ sections of each tissue were cut, washed in PBS and stained with hematoxylin for $5 \mathrm{~min}$ and eosin for $1 \mathrm{~min}$. One section following every 10th sections was chosen to be examined by a microscope (Leica TCS SP2 Microsystems, Heidelberg, Germany).

\section{Post-Occlusive Reactive Hyperemia (PORH) Responses of Skin Microvascular and Mesenteric Vascular Flow}

Rats were anesthetized and placed in a fixed prone position at a heated chamber, which was set at $37{ }^{\circ} \mathrm{C}$ to maintain constant temperature condition. Testing position was fixed on the hairless surface of the plantar paw in the right lower limb. Skin blood flow was measured using single-point laser Doppler flowmetry (LDF, Periflux System 5000, $780 \mathrm{~nm}$ laser diode, Perimed, Järfälla, Sweden) and the corresponding probe (Probe 407-1, the fiber spacing: $0.25 \mathrm{~mm}$, Perimed, Järfälla, Sweden) as previously described. ${ }^{26}$ After 3 min baseline recording, blood flow was occluded for 3 min by inflating an appropriately sized blood pressure cuff (Hokanson, Bellevue) placed around the upper hind leg to $200 \mathrm{~mm} \mathrm{Hg}$ to completely block the blood flow. The cuff was then released suddenly and data were recorded by the PeriSoft 2.5.5 program.

Similarly, after the small bowel was exposed and photographed, the mesenteric vascular responses to acetylcholine and sodium nitroprusside exposure were monitored. A section of the small intestines and mesentery was exteriorized, placed in a special shallow organ dish to reduce any movements because of respiration, bathed in $\mathrm{KHB}$ (at $37^{\circ} \mathrm{C}$, bubbled with $95 \% \mathrm{O}_{2}$ and $5 \% \mathrm{CO}_{2}$, and continuously replaced with fresh KHB using a pump). A probe of LDF was set on the fixed position of the mesentery with a rubber probe seat to record the blood flow variations in the mesenteric vessels. Acetylcholine and sodium nitroprusside (both $1 \mu \mathrm{mol} / \mathrm{l})$ were dissolved in KHB and delivered with Teflon tubing system attached to the laser Doppler probe $5 \mathrm{~mm}$ above the tissue as described previously. ${ }^{27}$ The responses to drugs were continuously recorded until its maximum effect on blood flow variations was reached.

\section{Western Blot}

Tissues and cells were lysed in RIPA buffer containing inhibitor cocktail (Santa Cruz Biotechnology, Santa Cruz) for $30 \mathrm{~min}$ on ice. Proteins were quantified using BCA assay kit. Lysates were dissolved on a 10\% SDS-PAGE gel and transferred to polyvinylidene difluoride membrane. Anti-eNOS, anti-phosphor eNOS (Ser1177) (Abcam), and $\alpha$-actin (Santa Cruz Biotechnology) antibodies were used at $4{ }^{\circ} \mathrm{C}$ overnight. The immunoblotted membrane was then incubated with horseradish peroxidase-conjugated secondary antibody for $1 \mathrm{~h}$ and immunoreactive bands were revealed using enhanced chemiluminescence assay (PerkinElmer Life Science Products). Relative luminescence intensity was analyzed by Quantity One software (Bio-Rad). 


\section{NO Measurements by ELISA}

NO level was determined by NO ELISA kit (BlueGene, Shanghai, China) in accordance with the manufacturer's protocol. For animal blood samples, we collected blood after rats were killed immediately using a serum separator tube, and allowed samples to clot for $1 \mathrm{~h}$ at room temperature. After centrifuged at $1000 \mathrm{~g}$ for $15 \mathrm{~min}$, the supernatant was harvested and determined for NO levels. For animal tissue homogenates, thoracic aortas, which were isolated from rats, were rinsed in ice-cold KHB to remove excess blood, weighed, then minced to small pieces, and homogenized in KHB. The resulting suspension was subjected to ultrasonication in order to further breaking the cell membranes. The homogenates were centrifuged at $1500 \mathrm{~g}$ for $15 \mathrm{~min}$. The supernatant was collected and assayed.

\section{Isolation and Culture of Adult Rat Cerebral Microvascular Endothelial Cells}

Cerebral microvascular endothelial cells were isolated from 4-week-old male Wistar rats as previously described. ${ }^{28}$ The primary cerebral microvascular endothelial cells were grown at $37^{\circ} \mathrm{C}$ in Endothelial Cell Medium (1001, ScienCell, USA) supplemented with $20 \%$ fetal bovine serum, $100 \mathrm{U} / \mathrm{ml}$ penicillin and $100 \mu \mathrm{g} / \mathrm{ml}$ streptomycin, and gassed with $5 \%$ $\mathrm{CO}_{2}$. The culture media were changed every 4 th day. Once reaching 90\% confluence, cells were digested and cultured until the 3rd passages and used. Endothelial cells were characterized by immunostaining with monoclonal antibody against the Cluster of Differentiation 31 adhesion molecule, a transmembrane glycoprotein expressed by endothelial cells. After starved with serum-free medium for $6 \mathrm{~h}$, cells were exposed to LMWF $(30 \mu \mathrm{g} / \mathrm{ml})$ and vehicle, respectively, for 10,20 , or $30 \mathrm{~min}$, then cultural media and cell lysates were collected.

\section{Data Analysis and Statistics}

Data are expressed as means \pm s.e.m. Multiple comparisons between treatment groups were made using a one-way
ANOVA followed by a LSD-test. Repeated ANOVA analysis was used for evaluation of concentration-response relation. The mesenteric lesion rates of different groups were compared using $\chi^{2}$ test with continuity correction or Fisher's exact test when the numbers of samples are less than 10 in each group. Two-sided significance tests were used throughout the data analysis, and $P<0.05$ was considered significant.

\section{RESULTS \\ Effect of LMWF on Development of Diabetes and Hypertension in GK Rats}

All of the diabetic GK rats at the age of 13-14 weeks showed greater hyperglycemic responses at indicated time points in oral glucose tolerance test before drug intervention (Figure 1a), and elevated non-fasting blood glucose and lowered body weight throughout the following 12 weeks of drug treatment compared with age-matched normal rats (Table 1). In addition, the blood pressure of the diabetic rats and blood lipids increased at the 4th week and rose further during the following 8 weeks when compared with normal rats (Table 1 and Figure 1b). LMWF at doses of 50, 100, and $200 \mathrm{mg} / \mathrm{kg} / \mathrm{day}$ significantly protected against diabetesinduced hypertension and hyperlipidemia, but not hyperglycemia or body weight loss. Probucol only ameliorated the elevated blood lipids; the other abnormalities remained unchanged in DM rats (Table 1 and Figure 1b). These data demonstrate that GK rats developed the characteristics of type 2 diabetes: abnormal blood glucose and lipid levels and higher basal hypertension. LMWF at all the three doses was able to prevent hyperlipidemia and hypertension but not the impaired glucose metabolism in vivo.

\section{Effects of LMWF on Endothelial Dysfunction and Mesentery Damage in Diabetic Rats}

To investigate whether LMWF has a protective effect on endothelial dysfunction in diabetic rats, shear stress-induced vasodilation, which is a physiological endothelium-dependent
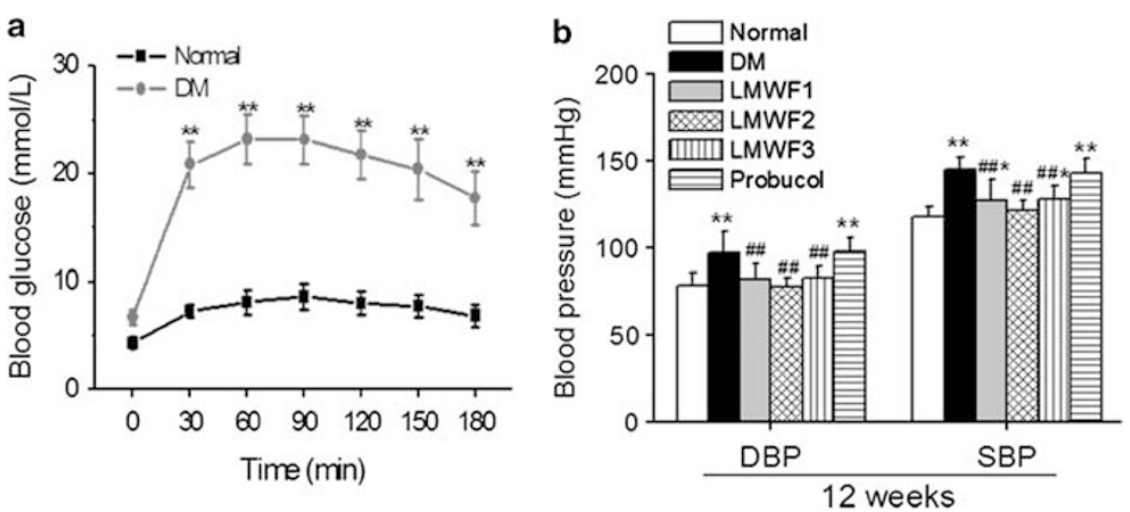

Figure 1 Effect of LMWF on basal hypertension in diabetic rats. (a) The oral glucose tolerance test (OGTT) was conducted on 13-week-old rats chosen at random between DM and normal groups, $n=10$ in each group. (b) The inhibitory effect of LMWF on basal hypertension after 12-week treatment, $n=8-15$ in each group. Data are expressed as mean \pm s.e.m. ${ }^{*} P<0.05,{ }^{* *} P<0.01$ vs normal group; ${ }^{\# \#} P<0.01$ vs DM group. 
Table 1 General parameters of normal Wistar and diabetic GK rats

\begin{tabular}{|c|c|c|c|c|c|c|}
\hline Groups (n) & Normal (15) & DM (15) & LMWF1 (12) & LMWF2 (12) & LMWF3 (8) & Probucol (10) \\
\hline \multicolumn{7}{|l|}{ Diastolic blood pressure $(\mathrm{mm} \mathrm{Hg})$} \\
\hline 8th week & $80.2 \pm 6.4$ & $90.5 \pm 8.9^{* *}$ & $83.7 \pm 6.5^{\# \#}$ & $80.6 \pm 7.4^{\# \#}$ & $85.3 \pm 8.4^{\#}$ & $92.4 \pm 7.1^{* *}$ \\
\hline 12th week & $78.6 \pm 7.1$ & $97.6 \pm 11.7^{* *}$ & $82.5 \pm 8.6^{\# \#}$ & $78.0 \pm 5.0^{\# \#}$ & $83.1 \pm 6.2^{\# \#}$ & $98.2 \pm 7.8^{* *}$ \\
\hline \multicolumn{7}{|l|}{ Systolic blood pressure $(\mathrm{mm} \mathrm{Hg})$} \\
\hline 4th week & $120.4 \pm 3.4$ & $127.4 \pm 7.6$ & $124.3 \pm 6.3$ & $125.1 \pm 3.3$ & $121.8 \pm 5.7$ & $122.1 \pm 2.4$ \\
\hline 8th week & $119.5 \pm 3.9$ & $138.1 \pm 8.9^{* *}$ & $124.7 \pm 9.9^{\#}$ & $120.3 \pm 5.4^{\# \#}$ & $125.7 \pm 6.9^{\#}$ & $133.8 \pm 6.7^{* *}$ \\
\hline 12th week & $117.8 \pm 6.1$ & $145.3 \pm 7.3^{* *}$ & $127.7 \pm 11.8^{* \# \#}$ & $121.6 \pm 5.8^{\# \#}$ & $128.3 \pm 7.7^{* \# \#}$ & $143.4 \pm 8.1^{* *}$ \\
\hline $\mathrm{CHO}(\mathrm{mmol} / \mathrm{l})$ & $1.78 \pm 0.25$ & $2.75 \pm 0.56^{* *}$ & $2.26 \pm 0.34^{* * \# \#}$ & $2.29 \pm 0.23^{* * \# \#}$ & $2.14 \pm 0.38^{\# \#}$ & $2.46 \pm 0.22^{* * \#}$ \\
\hline LDL-C (mmol/l) & $0.33 \pm 0.08$ & $0.51 \pm 0.18^{* *}$ & $0.33 \pm 0.10^{\# \#}$ & $0.29 \pm 0.04^{\# \#}$ & $0.29 \pm 0.08^{\# \#}$ & $0.34 \pm 0.05^{\# \#}$ \\
\hline $\mathrm{HDL}-\mathrm{C}(\mathrm{mmol} / \mathrm{l})$ & $1.26 \pm 0.23$ & $1.74 \pm 0.37^{* *}$ & $1.45 \pm 0.16^{\#}$ & $1.57 \pm 0.19^{* \#}$ & $1.35 \pm 0.28^{\# \#}$ & $1.56 \pm 0.16^{* \#}$ \\
\hline $\mathrm{TG}(\mathrm{mmol} / \mathrm{l})$ & $1.27 \pm 0.32$ & $1.44 \pm 0.29$ & $0.85 \pm 0.23^{* * \# \#}$ & $1.06 \pm 0.32^{\#}$ & $1.29 \pm 0.42$ & $1.29 \pm 0.29$ \\
\hline
\end{tabular}

Abbreviations: $\mathrm{CHO}$, cholesterol; $\mathrm{HDL}-\mathrm{C}$, high-density lipoprotein cholesterol; LDL-C, low-density lipoprotein cholesterol; TG, triglyceride.

Data are expressed as means \pm s.e.m. ${ }^{*} P<0.05 ;{ }^{* *} P<0.01$ vs normal group; ${ }^{\#} P<0.05$; ${ }^{\# \#} P<0.01$ vs diabetes (DM) group.

vessel relaxation response, was monitored by PORH method using laser Doppler flowmetry (see Materials and Methods). PORH refers to the flow-mediated increase in skin blood flow above baseline level after the release of an arterial occlusion and can be applied to rat paws as a comparative model for human skin blood flow. ${ }^{29}$ As shown in Figure 2a, the location of scanning area (indicated with a circle on the paw) was fixed in all the test animals. The relaxation function was evaluated by two parameters: the increase rate $=[$ peak flow $(\mathrm{PF})$ - resting flow $(\mathrm{RF})] / \mathrm{RF} \times 100 \%$ and the time required for the resting flow to reach after the occlusion released (TR). Diabetic rats exhibited much lower shear stress-induced vasodilation compared with normal rats, but diabetic rats treated with LMWF demonstrated an almost normal relaxation response upon release of the arterial occlusion. Probucol showed no effect in this test (Figures $2 \mathrm{~b}$ and $\mathrm{c}$ ).

Furthermore, we found obvious pathological changes in mesentery morphology in $80 \%$ of diabetic rats $(n=21)$, compared with the uniform mesentery and mesenteric vessels in normal rats $(n=30$, Figure $3 \mathrm{a})$. For comparison, this damage was relatively quantified into three degrees; mild (adipose aggregation and tissue adhesion), moderate (mild + arteriovenous malformation of mesenteric vessels), and severe (moderate + intestine necrosis). Proportions of $10 \%$ mild, 50\% moderate, and 20\% severe changes were seen in diabetic mesenteries, whereas only $30 \%, 15 \%$ and $20 \%$ mild but no moderate or severe damage were observed in diabetic rats treated with LMWF at dose of 50, 100, and $200 \mathrm{mg} / \mathrm{kg}$, respectively (Figure 3b). Probucol could decrease the incidence of pathological changes from 80 to $60 \%$, but the intensity of the lesions appeared unchanged compared with those in vehicle-treated diabetic rats.

Mesenteric damage in diabetes has been recognized as an inflammatory reaction because of endothelial dysfunction and deficiency of NO formation in mesenteric arteries. ${ }^{30-32}$ In addition, hypertrophy of medial layer in mesenteric artery with an accumulation of extracellular matrix proteins also contributes to the vessel change in GK rats. ${ }^{33}$ Thus, alterations in mesenteric artery function and contexture are a main cause of the pathogenesis of mesenteric lesions in diabetes. However, the mechanisms underlying the nature of the lesions and effectiveness of LMWF will require future studies.

Functional evaluation of mesenteric artery relaxation was further performed using laser Doppler flowmetry. In diabetic rats, the dilatory response to acetylcholine $(1 \mu \mathrm{mol} / \mathrm{l})$ disappeared, instead arterial constriction induced by acetylcholine-mediated activation of muscarinic receptor appeared (Figure 3d). As the relaxant responses of the mesenteric arteries to sodium nitroprusside $(1 \mu \mathrm{mol} / \mathrm{l})$ were similar among all the groups, these data indicate a damaged endothelium-dependent relaxation in diabetic mesenteric arteries. LMWF at all the three doses significantly improved 

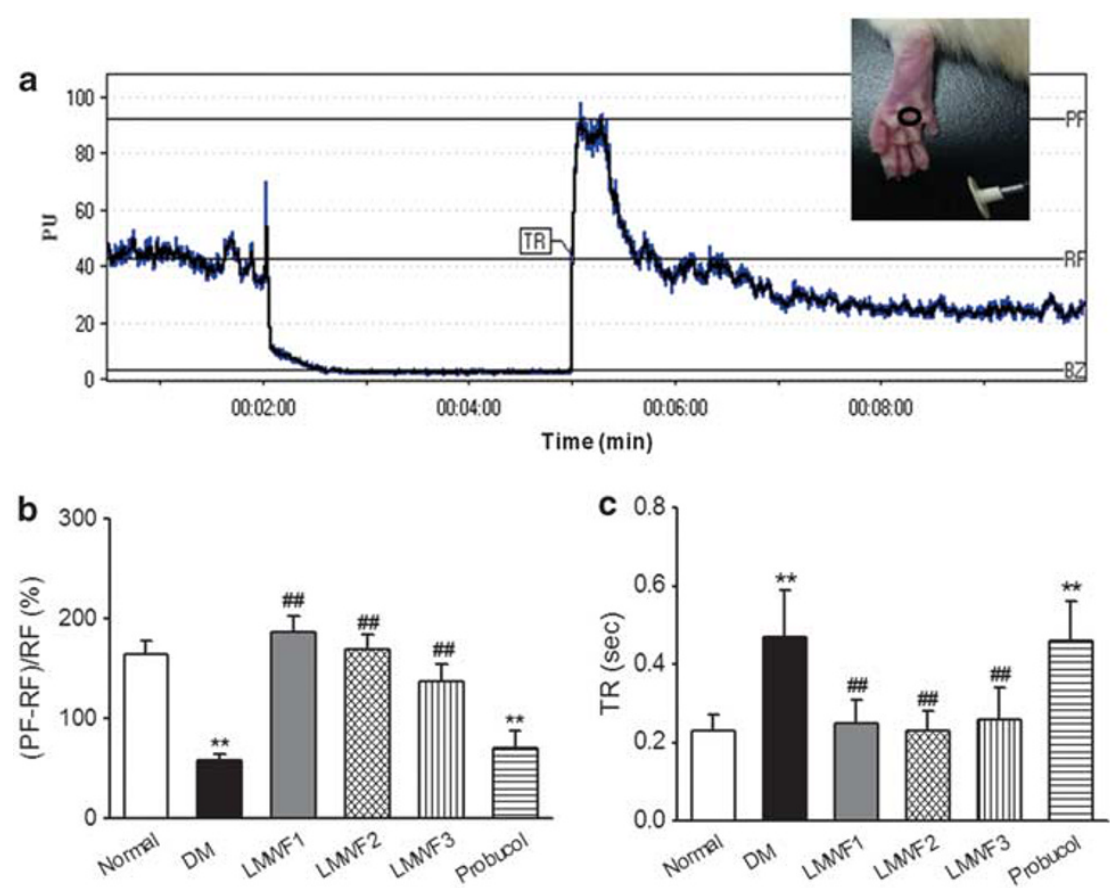

Figure 2 Effect of LMWF on flow-mediated endothelium-dependent dilation of plantar vessels in diabetic rats. The in vivo vasorelaxation was measured by laser Doppler flowmetry (see Materials and Methods). (a) The circle on the paw indicates the location where the probe was placed and as an example of PORH. PORH data were expressed as Biological Zero (BZ, blood flow when occluded), Peak Flow (PF, highest blood flow value after occlusion is released), Rest Flow (RF, normal blood flow), and Time to Recovery (TR, time required for the Rest Flow is reached after occlusion is released). (b, c) The statistical data of two parameters (PF $-\mathrm{RF}) / \mathrm{RF}$ and TR, for all groups of rats, $n=10$ in each group. ${ }^{*} P<0.01$ vs normal group; ${ }^{\# \# P}<0.01$ vs DM group.

the blood flow response of diabetic mesenteric vessel to acetylcholine, although $100 \mathrm{mg} / \mathrm{kg}$ LMWF was most effective. In contrast, probucol intervention produced no effect compared with the vehicle-treated GK rats.

\section{Effects of LMWF on Impaired Aortic Relaxation in Diabetic Rats}

To further identify the protective effect of LMWF on endothelium, hematoxylin and eosin staining sections of aortic rings were examined. Compared with normal aorta that displayed integral and successive endothelium, the aorta from diabetic group manifested uneven endangium with irregular thickness and partial absence of endothelium. LMWF treatment, especially at the dose of $100 \mathrm{mg} / \mathrm{kg}$, could ameliorate this pathological change in diabetic aorta (Figure 4a).

Functional examination demonstrated that the relaxation of phenylephrine $(1 \mu \mathrm{mol} / \mathrm{l})$-pre-contracted aortic rings by $10 \mu \mathrm{mol} / 1$ acetylcholine was much less in diabetic rats than that in normal rats, whereas the endothelium-independent relaxations to sodium nitroprusside were similar (Figure 4b). In consistent with the single concentration examination, the dose-responsible relaxation of aorta induced by accumulative addition of acetylcholine $\left(10^{-9}-10^{-5} \mathrm{~mol} / \mathrm{l}\right)$ was shifted to the right and maximal relaxation was suppressed, whereas the dose response to sodium nitroprusside remained unchanged in diabetic aorta. Diabetic rats treated with LMWF displayed improved responsiveness to accumulative acetylcholine exposure, whereas probucol treatment exhibited observable but not significant improvement (Figures $4 \mathrm{c}-\mathrm{e}$ ).

Therefore, all of the in vivo results demonstrate a protective effect of LMWF against diabetes-induced endothelial dysfunction and lesions in GK rats.

\section{Effects of LMWF on eNOS Expression and NO Production in Diabetic Rats}

It is well accepted that abnormal eNOS and NO bioavailability have important roles in endothelial dysfunction and lesions in diabetic subjects. ${ }^{3,7,10,11}$ Endothelium-derived $\mathrm{NO}$ is mainly generated by activation of eNOS and its phosphorylation at Ser1177. ${ }^{34,35}$ Thus, we next investigated the levels of eNOS expression and phosphorylation at Ser1177 in vessels and NO level in rat serum by western blotting and ELISA. As shown in Figure 5, the relative levels of eNOS expression, p-eNOS at Ser1177, and the NO synthesis were significantly decreased in diabetic aorta and also in caudal arteries (data not shown) compared with those in normal arteries. Consistent with the functional data (Figure 4), depressed eNOS expression and NO production because of diabetes were partially reversed in LMWF-treated diabetic rats; an almost complete recovery was seen in eNOS 
a
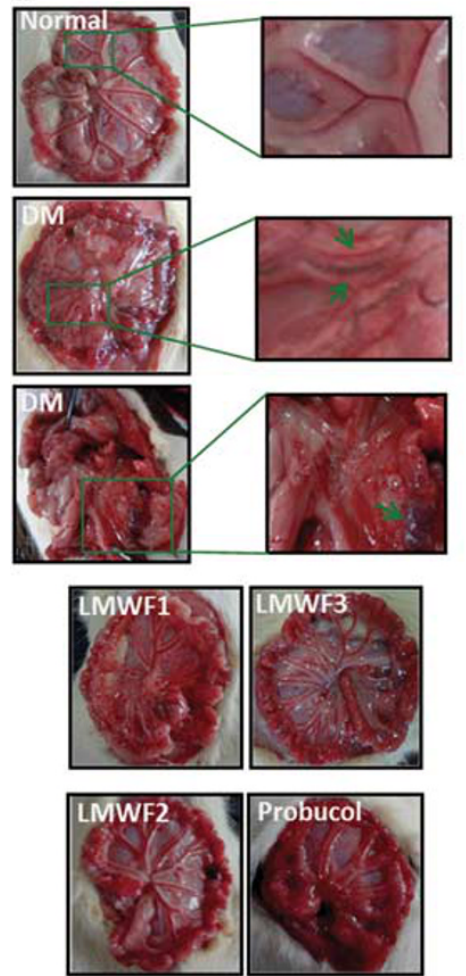

b

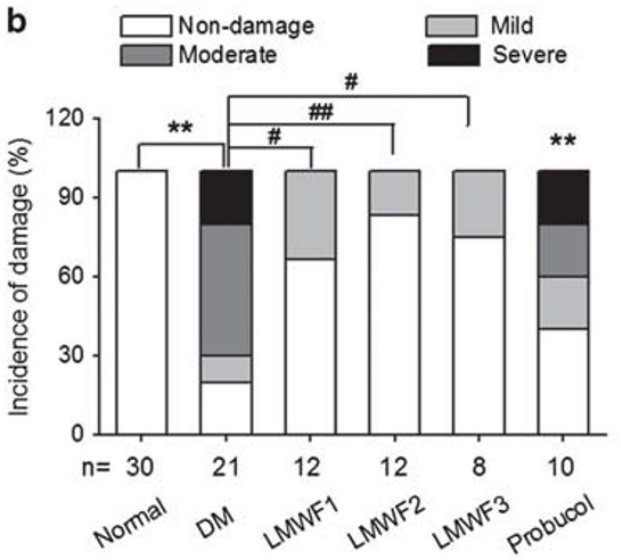

d

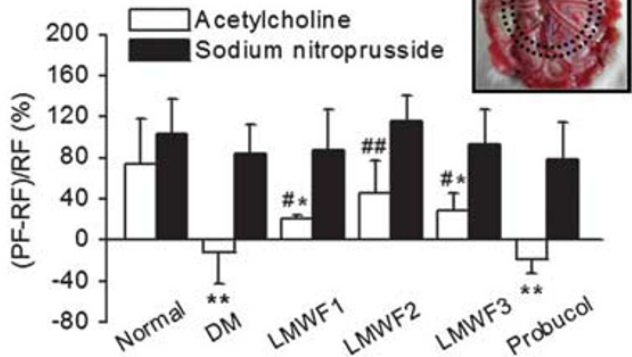

Figure 3 Effect of LMWF on damage and endothelial dysfunction of mesenteric vessels in diabetic rats. (a) Representative morphological pictures of mesentery in different groups of rats. (b) The incidence of mesenteric lesions is calculated in each group. (c) The sites circled by double dashed lines in the mesentery indicate the position where the probe was placed. (d) Statistical analysis of acetylcholine- $(1 \mu \mathrm{mol} / \mathrm{l})$ or sodium nitroprusside- $(1 \mu \mathrm{mol} / \mathrm{l})$ induced mesenteric vascular blood flow changes. $n=8-30$ in each group. ${ }^{*} P<0.05,{ }^{* *} P<0.01$ vs normal group; ${ }^{\#} P<0.05,{ }^{\# \#} P<0.01$ vs DM group.

phosphorylation at Ser1177 in diabetic rats that received $100 \mathrm{mg} / \mathrm{kg}$ LMWF. Probucol treatment produced an increase in eNOS expression but only a slight increase in Ser1177 phosphorylation and NO formation (Figure 5b).

\section{Effects of LMWF on eNOS Phosphorylation and NO Production in Normal Aorta and Cultured Vascular Endothelial Cells}

Finally, to further verify the effect of LMWF on eNOS, we conducted similar experiments in normal rat aorta and cultured microvascular endothelial cells isolated from rat cerebrum. We found that LMWF at concentrations of 6.25-50 $\mu \mathrm{g}$ / $\mathrm{ml}$ did not affect the baseline vasomotor and aortic constriction in response to an agonist in normal rats (data not shown), but significantly influenced the vessel relaxation of endothelial-intact aorta preconstricted by phenylephrine in a concentration-dependent manner. This relaxant effect was absent in aorta with denuded endothelium or in the presence of the NOS blocking agent L-NAME $\left(10^{-4} \mathrm{~mol} / \mathrm{l}\right.$; Figures $6 \mathrm{a}-\mathrm{c})$. Accordingly, LMWF $(25$ or $50 \mu \mathrm{g} / \mathrm{ml})$ treatment of aorta for $20 \mathrm{~min}$ induced robust eNOS phosphorylation at Ser1177 and a corresponding increase in NO production in a dose-dependent manner, whereas acetylcholine $(1 \mu \mathrm{mol} / \mathrm{l})$ elevated NO production without upregu- lation of Ser1177 phosphorylation (Figures 7a-c). These findings are consistent with the notion that acetylcholine mediates NO induction independent of Ser1177 phosphorylation. ${ }^{34,35}$

In cultured microvascular endothelial cells, treatment of the cells with LMWF at $30 \mu \mathrm{g} / \mathrm{ml}$ caused significant augmentations in Ser1177 phosphorylation and NO production, and their maximum responses were accordingly seen in the vasoendothelial cells exposure to LMWF for at least $20 \mathrm{~min}$ (Figures $7 \mathrm{~d}-\mathrm{f}$ ). These findings are consistent with the findings in LMWF-treated diabetic vessels (Figure 5) and normal aorta (Figures $7 \mathrm{a}-\mathrm{c}$ ).

\section{DISCUSSION}

This study demonstrates that GK diabetic rats developed hyperglycemia, hyperlipidemia, and impairment of vessels including elevated basal blood pressure, blunted endothelium-dependent vasorelaxation in response to either flow change or acetylcholine as well as lesions of mesentery and aortic endangium. In addition, deficient eNOS phosphorylation at Ser1177 and reduced eNOS expression and NO production also occurred in GK rats, demonstrating typical impairment of vasoendothelium in type 2 diabetes. $^{1-5}$ Importantly, the present study demonstrates that treatment 

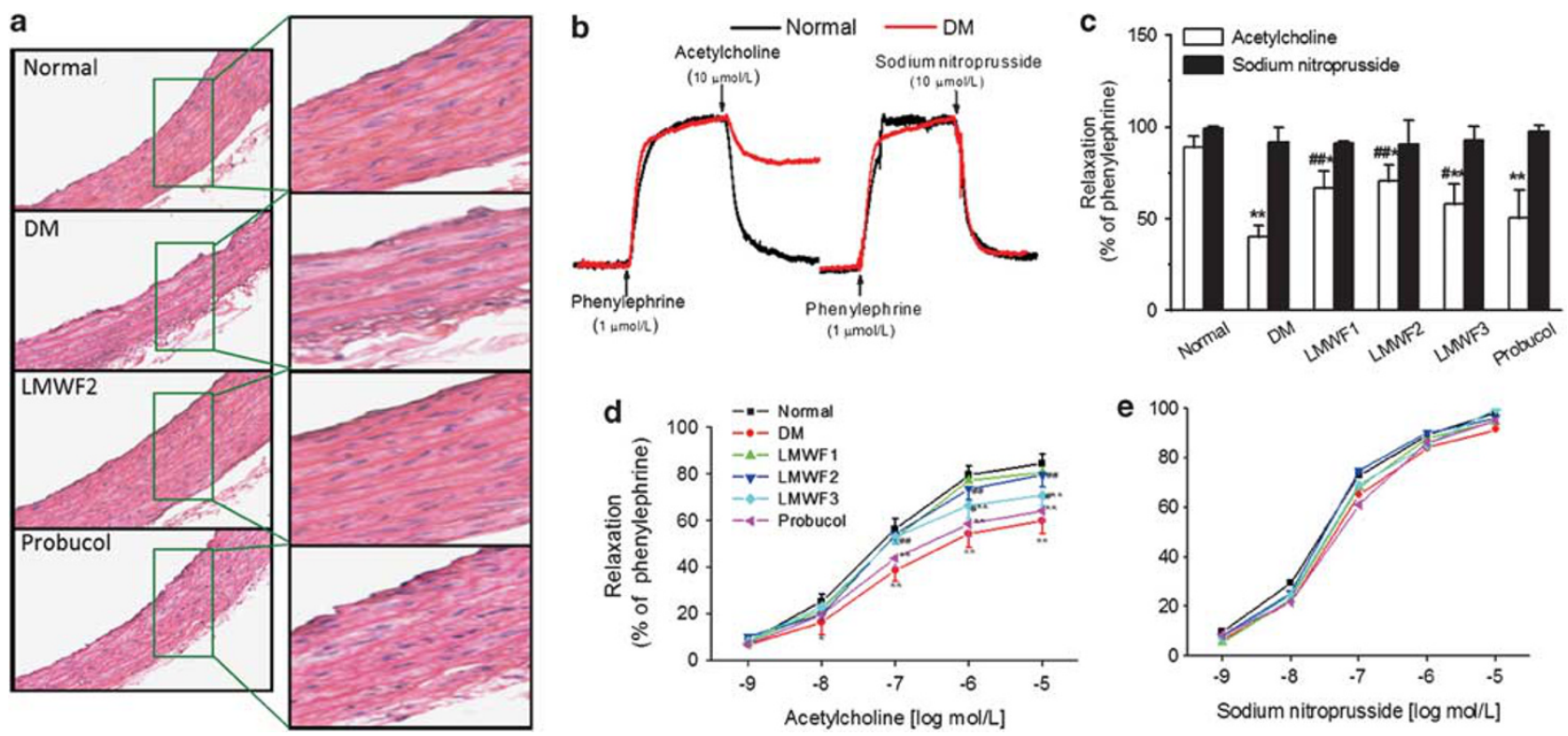

Figure 4 Effect of LMWF on the impaired endothelium-dependent relaxation in aorta of diabetic rats. (a) Hematoxylin and eosin (H-E) staining $(\times 20)$ of aortic sections from normal, DM, LMWF2, and probucol group. (b) Representative tracings of vessel tension in response to acetylcholine- (10 $\mu$ mol/l) and sodium nitroprusside- $(10 \mu \mathrm{mol} / \mathrm{l})$ induced vasodilation in arterial rings pre-contracted with phenylephrine $(1 \mu \mathrm{mol} / \mathrm{l})$ between normal and DM group, respectively. (c) A single dose $(10 \mu \mathrm{mol} / \mathrm{l})$ of acetylcholine- or sodium nitroprusside-induced vasodilation in each group. (d, e) Cumulative-doses $\left(10^{-9}-10^{-5} \mathrm{~mol} / \mathrm{l}\right)$ acetylcholine- or sodium nitroprusside-induced vasodilation in each group. Endothelium-dependent vascular relaxation was impaired in DM group $(P<0.01$ vs normal group), whereas endothelium-independent relaxation was unaltered. LMWF improved endotheliumdependent vascular relaxation. $n=12-15$ segments in each group, two vascular rings per animal. ${ }^{*} P<0.05$, ${ }^{* *} P<0.01$ vs normal group; ${ }^{\#} P<0.05$, \#\# $P<0.01$ vs DM group.
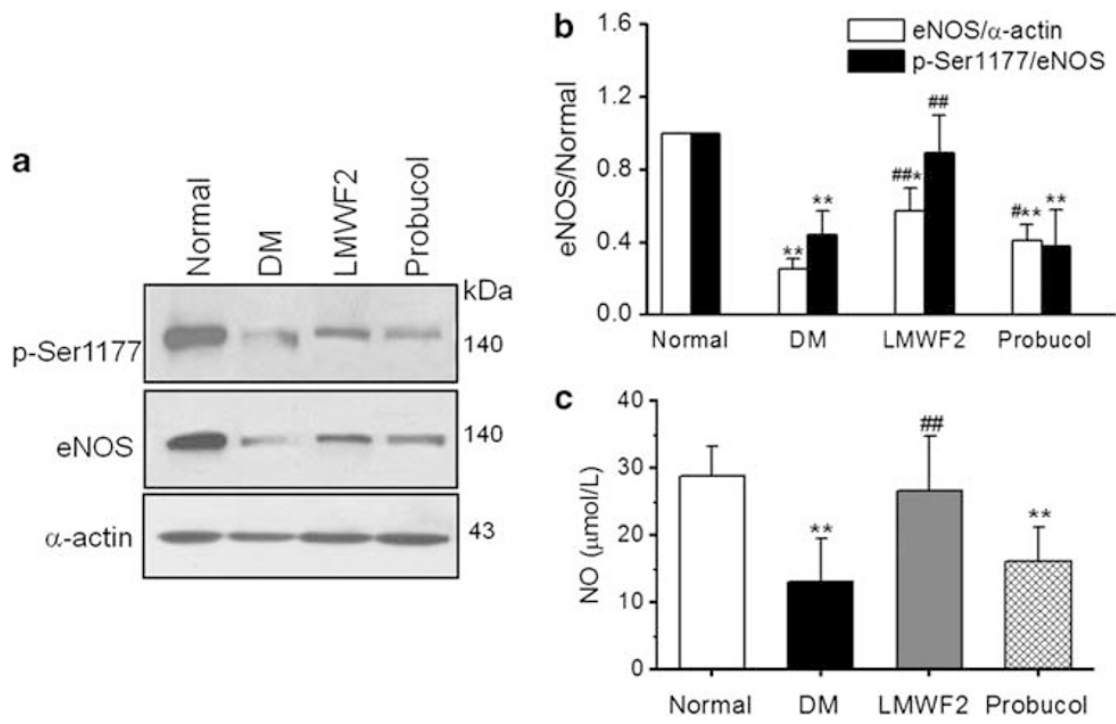

Figure 5 Effect of LMWF on the impaired eNOS expression and phosphorylation at Ser1177. (a, b) Protein expressions of total eNOS and p-eNOS at Ser1177 in aortic tissues from normal, DM, LMWF2, and probucol rats. (c) LMWF increased serum NO levels in diabetic rats. $n=6$ in each group. ${ }^{*} P<0.05,{ }^{*} P<0.01$ vs normal group; ${ }^{\#} P<0.05,{ }^{\# \#} P<0.01$ vs DM group.

of GK rats with LMWF profoundly ameliorated these diabetes-associated disorders, based on the observations that (1) LMWF improved the basal blood pressure and shear stress-induced vasodilation, and protected mesentery integrity in diabetic rats; (2) LMWF ameliorated the pathological changes in endangium and the endothelium-dependent vasodilation, and upregulated NO synthesis in diabetic rats; and (3) mechanistically, LMWF induced an endothelium/ eNOS/NO-dependent vasorelaxation, in particular an enhancement of eNOS phosphorylation at Ser1177 in both 
a

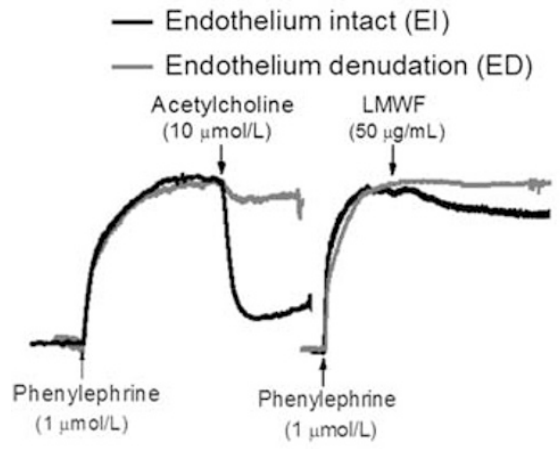

b

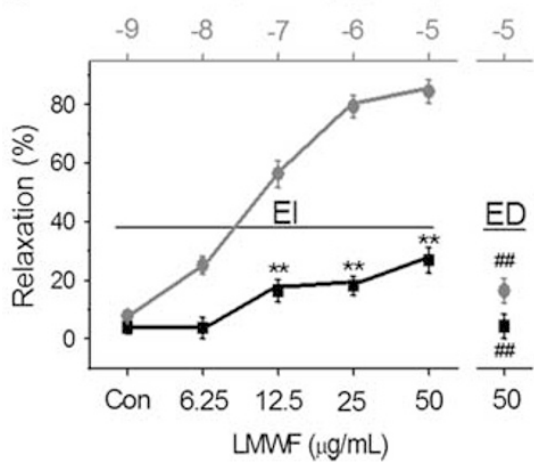

c

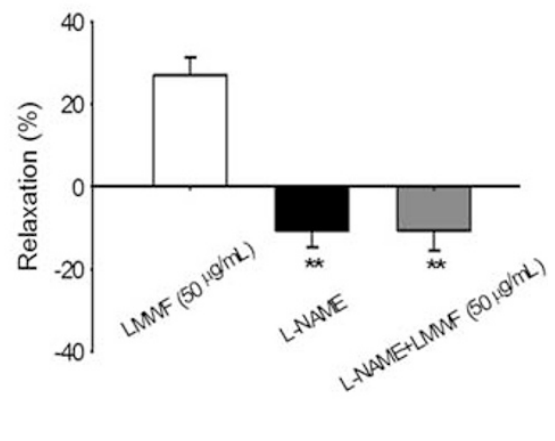

Figure 6 Effect of LMWF on endothelium-dependent dilation in normal aorta. Vessels were pre-contracted with $1 \mu$ mol/l phenylephrine. (a) Representative tracings of vessel tension of intact endothelium and denuded endothelium in aorta rings after treatment with acetylcholine and LMWF, respectively. (b) Aorta ring relaxation in response to cumulative concentrations of acetylcholine and LMWF in intact and denuded endothelium. ${ }^{*} P<0.05,{ }^{* *} P<0.01$ vs Con; ${ }^{\# \#} P<0.01$ denuded endothelium + LMWF $(50 \mu \mathrm{g} / \mathrm{ml})$ vs intact endothelium + LMWF (50 $\left.\mu \mathrm{g} / \mathrm{ml}\right)$. (c) Statistical analysis of aorta ring relaxation in the presence or absence of LMWF $(50 \mu \mathrm{g} / \mathrm{ml})$ after incubation with L-NAME $\left(10^{-4} \mathrm{~mol} / \mathrm{l}\right)$ for $30 \mathrm{~min}$. ${ }^{* *} P<0.01 \mathrm{vs}$ LMWF $(50 \mu \mathrm{g} / \mathrm{ml}) . n=12-15$ segments in each group.

non-diabetic and diabetic aorta and in cultured vascular endothelial cells.

Physiologically, endothelium has a fundamental role in maintenance of vascular function and homeostasis. ${ }^{7-9}$ Under pathological circumstances, such as high glucose, hypertension, or oxidative stress, endothelial dysfunction characterized by a reduction in NO bioavailability is recognized as the hallmark of atherosclerotic diseases and prediction of cardiovascular events. ${ }^{4,5,7}$ Because of the multiple risk factors coexisting in diabetes, diabetes patients are more susceptible to endothelial dysfunction and lesions, and eventually suffer cardiovascular complications. eNOS has been identified as the enzyme responsible for most of the $\mathrm{NO}$ production in vasculature, and it may undergo a harmful change in its enzymology termed 'eNOS uncoupling', ie, converting from formation of $\mathrm{NO}$ to generating $\mathrm{O}_{2}{ }^{-}$that further consumes $\mathrm{NO}$, making toxic peroxynitrite $\left(\mathrm{ONOO}^{-}\right)$in diabetes. ${ }^{7-10}$ Concomitantly, reduced eNOS expression and NO release may also take place, particularly in persistently stimulated and injured endothelium. ${ }^{2,7,9}$ Therefore, functional loss and impaired expression of eNOS in diabetes have major pathophysiologic consequences to vascular function and health. . $^{2,8}$

In addition to its well-recognized anti-inflammatory, antioxidative, anticoagulative, and antiaggregative effects, fucoidan also inhibits vascular smooth muscle cell migration and proliferation. ${ }^{36,37}$ In addition, it blocks selectin-induced leukocyte and platelet accumulation to ischemic artery and prevent further damage and thrombosis. ${ }^{38,39}$ In isolated endothelial cells, fucoidan is found to block the formation of capillary-like structures (tubes) by the endothelial cells, ${ }^{14}$ inhibit adhesion of sickle erythrocytes to endothelium, ${ }^{40}$ and attenuate endothelium-mediated angiogenesis. ${ }^{41}$ Moreover, fucoidan also modulates proliferation and migration of human endothelial cells, suggesting a potential role of fucoidan as a new therapeutic agent for vascular endothelial damage. ${ }^{14,42}$ LMWF is the fragment of unfractionated fucoidan obtained by either chemical or enzymatic depolymerization. In comparison to native fucoidan, LMWF possesses a higher degree of bioactivity. ${ }^{14,15}$ Previously, we compared two preparations of fucoidans $(\sim 120$ and $7 \mathrm{kDa})$ extracted from edible species of L. japonica and commercially available fucoidan $(50 \mathrm{kDa})$ and found that LMWF $(7 \mathrm{kDa})$ exerts an antithrombotic effect without impacting coagulation, whereas the others affect coagulation at the concentration that inhibits platelet aggregation. ${ }^{19}$ In addition, it has obvious protective effects against brain inflammation and renal ischemia-reperfusion injury. ${ }^{17,18}$ Here we demonstrated that LMWF corrected the impaired endothelium-dependent vasorelaxation and eNOS function in diabetic rats. Importantly, these effects of LMWF are through the protections of eNOS phosphorylation at Ser1177 residue and its expression in vasoendothelium, highlighting its effectiveness on dysfunctional endothelium, and thus anti-hypertension in diabetic animals. Although both probucol and LMWF show similar anti-inflammatory and anti-oxidative effects, and improve hyperlipidemia, probucol only exerted a slight effect on impaired endothelium-dependent relaxation in GK rats, which is in line with the observations in rat models of type 1 diabetes. ${ }^{43}$ The benefit of probucol on cardiovascular complication in diabetes is still controversial, although multiple studies show an obvious recovery from diabetic kidney damage by probucol. ${ }^{44,45}$ The vasoprotective effect of LMWF in type 2 diabetes is more significant than probucol in this study, reflecting the importance of maintaining eNOS phosphorylation and $\mathrm{NO}$ production in protection of the vasoendothelium of diabetes.

The primary eNOS phosphorylation sites are serine residues, but, to a lesser extent, tyrosine and threonine 

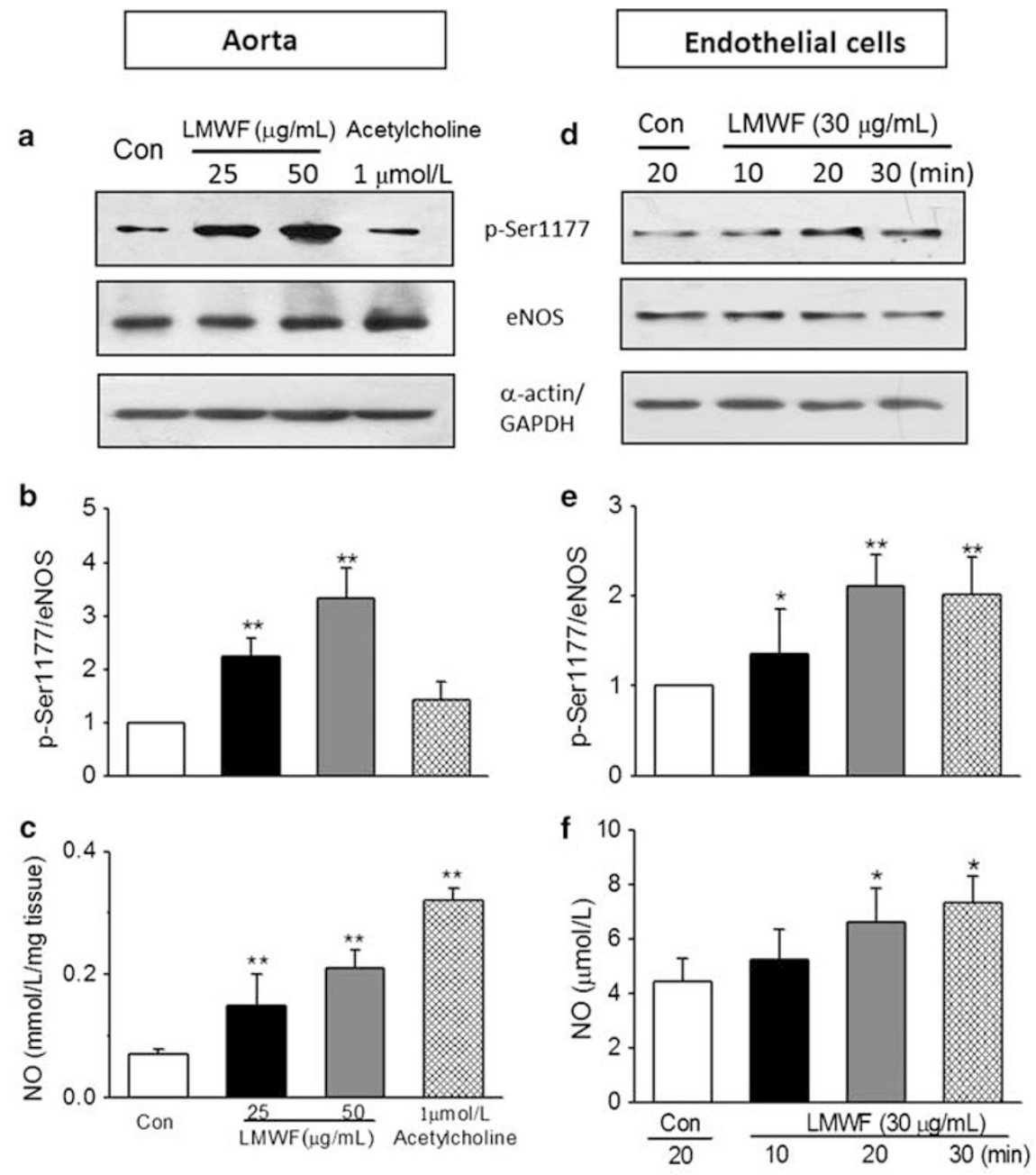

Figure 7 Effect of LMWF on eNOS and p-eNOS at Ser1177 in aorta and vasoendothelial cells. Western blots of total eNOS and p-eNOS at Ser1177, and the measurement of NO production in thoracic arterial rings of normal rat and cultured microvascular endothelial cells. (a-c) Protein expression of total eNOS and p-eNOS at Ser1177, and NO production in the thoracic arterial rings after incubation with acetylcholine $(1 \mu \mathrm{mol} / \mathrm{l})$ and LMWF $(25 \mu \mathrm{g} / \mathrm{ml}$ or $50 \mu \mathrm{g} / \mathrm{ml}$ ) for $30 \mathrm{~min} . n=6$ segments in each group, ${ }^{*} P<0.05,{ }^{*} P<0.01$ vs Con. (d $\left.-\mathbf{f}\right)$ Protein expressions of eNOS and p-eNOS at Ser1177, and NO production in cultured microvascular endothelial cells after incubation with LMWF ( $30 \mu \mathrm{g} / \mathrm{ml})$ for 10,20 , and $30 \mathrm{~min}$. $n=5$ in each group. ${ }^{* *} P<0.01 \mathrm{vs}$ control $20 \mathrm{~min}$

phosphorylation can also occur. The most extensively studied eNOS phosphorylation site is Ser1177, as diverse stimuli that induce eNOS activation cause eNOS phosphorylation at this residue. $^{34,46}$ Akt, AMP-activated protein kinase (AMPK), $\mathrm{Ca}^{2+} /$ calmodulin-dependant protein kinase, and protein kinase A are potentially involved in the mediation of eNOS-Ser1177 phosphorylation. ${ }^{46,47}$ Thus, in this study, we also investigated the possible pathway(s) for the upregulation of eNOS phosphorylation at Ser1177 by LMWF, and found that no significant changes were observed in the levels of Akt and ERK in vasoendothelial cells treated with LMWF (data not shown). In addition, Jeong et al found that LMWF improves insulin sensitivity through AMPK activation and restores lipid homeostasis in a mouse model of type 2 diabetes. ${ }^{48}$ Thus, this may be one of the mechanisms that explain its activation of eNOS phosphorylation; however, further and more detailed studies are necessary regarding how LMWF improves endothelial dysfunction in diabetes. In addition, it appears that LMWF demonstrated a dosedependent but not proportional effect on endothelium damage in diabetic rats. This may be due to the differences in body systematic $v s$ tissue (cell) local treatment and response toward LMWF or multifactor integrated damage in endothelium in the setting of diabetes $v s$ normal endothelium and vasoendothelial cells.

In summary, diabetic cardiovascular complications are a burden worldwide. Endothelial dysfunction, characterized by impairment of eNOS and NO bioavailability, has been implicated in the pathogenesis of diabetic cardiovascular complications. Multiple drugs that target at different 
points of pathogenesis, including protection of endothelial function, are strongly recommended for the treatment of diabetes..$^{811-13}$ Here, we showed the protective effect of LMWF on vascular endothelial function through its maintenance of eNOS function and NO production in diabetic GK rats. LMWF also possesses additional beneficial effects such as antioxidative and anti-inflammatory properties, and appears to be a safer treatment option. ${ }^{49}$ Taken together, our data provide the first evidence for LMWF as a candidate drug for prevention and treatment of diabetic cardiovascular complications.

\section{ACKNOWLEDGMENTS}

This study was supported by grants from Beijing Natural Science Foundation (5122006), National Natural Science Foundation (81370339, 30973537) and Beijing Key Laboratory of Metabolic Disorders Related Cardiovascular Diseases (Z13111000280000).

\section{DISCLOSURE/CONFLICT OF INTEREST}

The authors declare no conflict of interest.

1. Sowers JR, Epstein M, Frohlich ED. Diabetes, hypertension, and cardiovascular disease: an update. Hypertension 2001;37:1053-1059.

2. Brownlee M. Biochemistry and molecular cell biology of diabetic complications. Nature 2001;414:813-820.

3. Forstermann U, Munzel T. Endothelial nitric oxide synthase in vascular disease: from marvel to menace. Circulation 2006;113:1708-1714.

4. Kim J, Montagnani M, Koh KK, et al. Reciprocal relationships between insulin resistance and endothelial dysfunction: molecular and pathophysiological mechanisms. Circulation 2006;113:1888-1904.

5. Maskrey BH, Megson IL, Whitfield PD, et al. Mechanisms of resolution of inflammation: a focus on cardiovascular disease. Arterioscler Thromb Vasc Biol 2011;31:1001-1006.

6. Spinetti G, Kraenkel N, Emanueli C, et al. Diabetes and vessel wall remodelling: from mechanistic insights to regenerative therapies. Cardiovasc Res 2008;78:265-273.

7. Esper RJ, Nordaby RA, Vilarino JO, et al. Endothelial dysfunction: a comprehensive appraisal. Cardiovasc Diabetol 2006;5:4.

8. Sandra JH, Gerard TC, Gerald FW. Therapeutic regulation of endothelial dysfunction in type 2 diabetes mellitus. Diabetes Vasc Dis Res 2007:4:89-102.

9. Sena CM, Nunes E, Louro $T$, et al. Effects of alpha-lipoic acid on endothelial function in aged diabetic and high-fat fed rats. $\mathrm{Br} J$ Pharmacol 2008;153:894-906.

10. Bouloumié A, Bauersachs J, Linz W, et al. Endothelial dysfunction coincides with an enhanced NO synthase expression and superoxide anion production. Hypertension 1997;30:934-941.

11. Hink $\mathrm{U}, \mathrm{Li} \mathrm{H}, \mathrm{Mollnau} \mathrm{H}$, et al. Mechanisms underlying endothelial dysfunction in diabetes mellitus. Circ Res 2001;88:14-22.

12. Verspohl EJ. Novel Pharmacological approaches to the treatment of type 2 diabetes. Pharmacol Rev 2012;64:188-237.

13. Ceriello $\mathrm{A}$, Kumar $\mathrm{S}$, Piconi $\mathrm{L}$, et al. Simultaneous control of hyperglycemia and oxidative stress normalizes endothelial function in type 1 Diabetes. Diabetes Care 2007;30(Suppl 3):649-654.

14. Cumashi A, Ushakova NA, Preobrazhenskaya ME, et al. A comparative study of the anti-inflammatory, anticoagulant, antiangiogenic, and antiadhesive activities of nine different fucoidans from brown seaweeds. Glycobiology 2007;17:541-552.

15. Richard B, Bouton MC, Loyau S, et al. Modulation of protease nexin-1 activity by polysaccharides. Thromb Haemost 2006;95:229-235.

16. Wang J, Zhang $Q$, Zhang $Z$, et al. Potential antioxidant and anticoagulant capacity of low molecular weight fucoidan fractions extracted from Laminaria japonica. Int J Biol Macromol 2010;46:6-12.

17. Luo DZ, Zhang $\mathrm{QB}$, Wang $\mathrm{XM}$. Fucoidan protects against dopaminergic neuron death in vivo and in vitro. Eur J Pharmacol 2009;617:33-41.
18. Chen J, Wang W, Zhang Q, et al. Low molecular weight fucoidan against renal ischemia-reperfusion injury via inhibition of the MAPK signaling pathway. PLoS One 2013;8:e56224.

19. Zhu Z, Zhang $Q$, Chen $L$, et al. Higher specificity of the activity of low molecular weight fucoidan for thrombin-induced platelet aggregation. Thromb Res 2010;125:419-426.

20. Cheng ZJ, Vaskonen T, Tikkanen I, et al. Endothelial dysfunction and salt-sensitive hypertension in spontaneously diabetic Goto-Kakizaki rats. Hypertension 2001;37:433-439.

21. Galli J, Fakhrai-Rad H, Kamel A, et al. Pathophysiological and genetic characterization of the major diabetes locus in GK rats. Diabetes 1999:48:2463-2470.

22. Beck K, Wu B J, Ni J, et al. Interplay between heme oxygenase-1 and the multifunctional transcription factor Yin Yang 1 in the inhibition of intimal hyperplasia. Circ Res 2010;107:1490-1497.

23. Kim SS, Gallaher DD, Csallany AS. Vitamin E and probucol reduce urinary lipophilic aldehydes and renal enlargement in streptozotocininduced diabetic rats. Lipids 2000;35:225-1237.

24. Nomura S, Takahash N, Inamii N, et al. Probucol and ticlopidine: effect on platelet and monocyte activation markers in hyperlipidemic patients with and without type 2 diabetes. Atherosclerosis 2004;174: 329-335.

25. Clark JD, Baldwin RL, Bayne KA, et al. Guide for the care and use of laboratory animals. Institute of Laboratory Animal Resources, National Research Council: Washington, DC, 1996.

26. Capp CL, Dorwart WC, Elias NT, et al. Post pressure hyperemia in the rat. Comp Biochem Physiol A Mol Integr Physiol 2004;137:533-546.

27. Vukosavljevic N, Jaron D, Barbee KA, et al. Quantifying the L-arginine paradox in vivo. Microvasc Res 2006;71:48-54.

28. Ichikawa $\mathrm{N}$, Naora $\mathrm{K}$, Hirano $\mathrm{H}$, et al. Isolation and primary culture of rat cerebral microvascular endothelial cells for studying drug transport in vitro. J Pharmacol Toxicol Methods 1996;36:45-52.

29. Rendell MS, McIntyre SF, Terando JV, et al. Skin blood flow in the Wistar-Kyoto rat and the spontaneously hypertensive rat. Comp Biochem Physiol Comp Physiol 1993;106:349-354.

30. Chatterjee TK, Stoll LL, Denning GM, et al. Proinflammatory phenotype of perivascular adipocytes: influence of high-fat feeding. Circ Res 2009;104:541-549.

31. Zhang $\mathrm{H}$, Zhang C. Adipose "talks" to distant organs to regulate insulin sensitivity and vascular function. Obesity (Silver Spring) 2010;18:20712076.

32. Bohlen HG, Lash JM. Topical hyperglycemia rapidly suppresses EDRF-mediated vasodilation of normal rat arterioles. Am J Physiol 1993:265:219-225.

33. Song W, Ergu A. Type-2 diabetes-induced changes in vascular extracellular matrix gene expression: Relation to vessel size. Cardiovascular Diabetology 2006;5:3.

34. Mount PF, Kemp BE, Power DA. Regulation of endothelial and myocardial NO synthesis by multi-site eNOS phosphorylation. J Mol Cell Cardiol 2007:42:271-279.

35. Kolluru GK, Siamwala JH, Chatterjee S. eNOS phosphorylation in health and disease. Biochimie 2010;92:1186-1198.

36. Deux JF, Anne MP, Alain F, et al. Low molecular weight fucoidan prevents neointimal hyperplasia in rabbit iliac artery in-stent restenosis model. Arterioscler Thromb Vasc Biol 2002;22:1604-1609.

37. Patel MK, Mulloy B, Gallagher KL, et al. The antimitogenic action of the sulphated polysaccharide fucoidan differs from heparin in human vascular smooth muscle cells. Thromb Haemost 2002;87:149-154.

38. Chauvet $\mathrm{P}$, Bienvenu JG, Théorêt JF, et al. Inhibition of plateletneutrophil interactions by Fucoidan reduces adhesion and vasoconstriction after acute arterial injury by angioplasty in pigs. J Cardiovasc Pharmacol 1999;34:597-603.

39. Barrabes JA, Garcia-Dorado D, Mirabet $M$, et al. Antagonism of selectin function attenuates microvascular platelet deposition and plateletmediated myocardial injury after transient ischemia. J Am Coll Cardiol 2005;45:293-299.

40. Barabino GA, Liu XD, Ewenstein BM, et al. Anionic polysaccharides inhibit adhesion of sickle erythrocytes to the vascular endothelium and result in improved hemodynamic behavior. Blood 1999;93: $1422-1429$.

41. Narazaki M, Segarra M, Tosato G. Sulfated polysaccharides identified as inducers of neuropilin-1 internalization and functional inhibition of VEGF 165 and semaphorin3A. Blood 2008;111:4126-4136. 
42. Giraux JL, Matou S, Bros A, et al. Modulation of human endothelial cell proliferation and migration by fucoidan and heparin. Eur J Cell Biol 1998;77:352-359.

43. Palmer AM, Gopaul N, Dhir S, et al. Endothelial dysfunction in streptozotocin-diabetic rats is not reversed by dietary probucol or simvastatin supplementation. Diabetologia 1998;41:157-164.

44. Agardh E, Hultberg B, Agardh C. Effects of inhibition of glycation and oxidative stress on the development of cataract and retina vessel abnormalities in diabetic rats. Curr Eye Res 2000;21: 543-549.

45. Tanous D, Hime N, Stocker R. Anti-atherosclerotic and anti-diabetic properties of probucol and related compounds. Redox Rep 2008, 13:48-59.
46. Dimmeler S, Fleming I, Fisslthaler B, Hermann C, et al. Activation of nitric oxide synthase in endothelial cells by Akt-dependent phosphorylation. Nature 1999;399:601-605.

47. Forstermann U, Sessa WC. Nitric oxide synthases: regulation and function. Eur Heart J 2012;33:829-837.

48. Jeong YT, Kim YD, Jung YM, et al. Low molecular weight fucoidan improves endoplasmic reticulum stress-reduced insulin sensitivity through AMP-activated protein kinase activation in L6 myotubes and restores lipid homeostasis in a mouse model of type 2 diabetes. Mol Pharmacol 2013;84:147-157.

49. Li N, Zhang Q, Song J. Toxicological evaluation of fucoidan extracted from Laminaria japonica in Wistar rats. Food Chem Toxicol 2005; 43:421-426. 